\title{
Suppression of isotopic polymorphism
}

\author{
Nicholas P. Funnell, David R. Allan, Andrew G. P. Maloney, Ronald \\ I. Smith, Cameron J. G. Wilson and Simon Parsons
}

\section{Published version information}

Citation: NP Funnell et al. 'Suppression of isotopic polymorphism.' CrystEngComm, vol. 23, no. 4 (2021): 769-776.

DOI: $10.1039 /$ d0ce01636e

This version is made available in accordance with publisher policies. Please cite only the published version using the reference above. This is the citation assigned by the publisher at the time of issuing the AAM. Please check the publisher's website for any updates. 


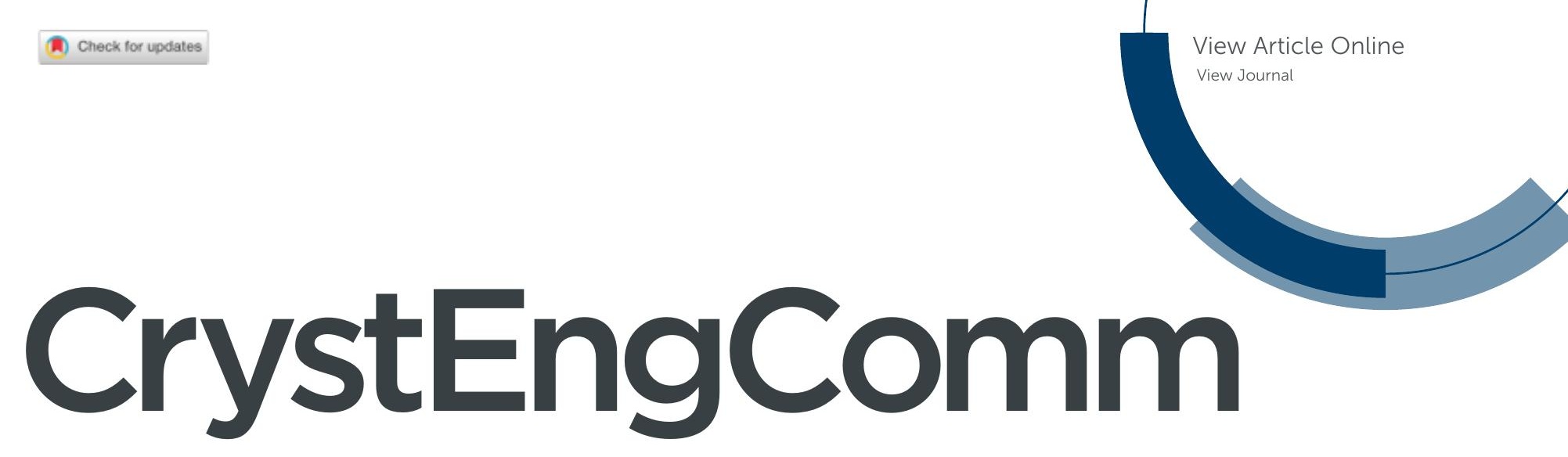

\section{Accepted Manuscript}

This article can be cited before page numbers have been issued, to do this please use: N. P. Funnell, D. R. Allan, A. G. P. Maloney, R. Smith, C. J. G. Wilson and S. Parsons, CrystEngComm, 2021, DOI:

\subsection{9/D0CE01636E.}
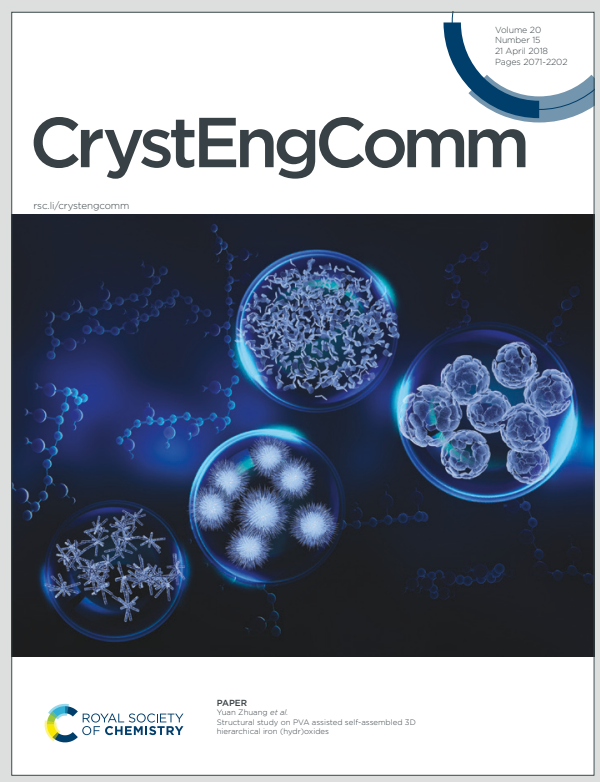

This is an Accepted Manuscript, which has been through the Royal Society of Chemistry peer review process and has been accepted for publication.

Accepted Manuscripts are published online shortly after acceptance, before technical editing, formatting and proof reading. Using this free service, authors can make their results available to the community, in citable form, before we publish the edited article. We will replace this Accepted Manuscript with the edited and formatted Advance Article as soon as it is available.

You can find more information about Accepted Manuscripts in the Information for Authors.

Please note that technical editing may introduce minor changes to the text and/or graphics, which may alter content. The journal's standard Terms \& Conditions and the Ethical guidelines still apply. In no event shall the Royal Society of Chemistry be held responsible for any errors or omissions in this Accepted Manuscript or any consequences arising from the use of any information it contains. 


\title{
Journal Name
}

\section{ARTICLE TYPE}

Cite this: DOI: 00.0000/xxxxxxxxxx

\section{Suppression of isotopic polymorphism ${ }^{\dagger}$}

\author{
Nicholas P. Funnell, ${ }^{a, *}$ David R. Allan, ${ }^{b}$ Andrew G. P. Maloney, ${ }^{c}$ Ronald I. Smith, ${ }^{a}$ \\ Cameron J. G. Wilson ${ }^{d}$ and Simon Parsons ${ }^{d, *}$
}

Received Date

Accepted Date

DOI: $00.0000 / x x x x x x x x x x$

The 4-methylpyridine pentachlorophenol co-crystal is known to exhibit the unusual phenomenon of isotopic polymorphism - the hydrogenated form crystallises with triclinic symmetry whereas deuteration of the phenol leads to growth of a monoclinic form instead. We report the use of pressure to overcome the zero point energy difference that leads to the two forms, instead engineering the formation of a hydrogenated, monoclinic co-crystal. This form cannot be obtained via direct compression of the triclinic form, but can be recovered from a diamond anvil cell following pressure-induced growth from solution. Furthermore, with Density Functional Theory calculations, we outline the conditions under which the remaining triclinic-deuterated form might be obtained.

\section{Introduction}

The substitution of hydrogen for deuterium in molecules is commonly performed, for example, when there is a need to contrast between atomic sites in ${ }^{1} \mathrm{H}$ NMR measurements or to avoid undesirable incoherent scattering when using neutron radiation. The assumption is often made that the structural behaviour of compounds and their crystal structures is unaffected by this exchange. While in many cases this assumption holds true there is a small, but increasing, number of materials where deuteration is known to affect their solid-state structures more profoundly. ${ }^{1,2}$ There are several examples of phase boundaries shifting with isotope substitution, ${ }^{3-5}$ and perturbations to atomic coordinates, ${ }^{6}$ but more significant is the formation of entirely new crystalline phases; socalled 'isotopic polymorphism'. ${ }^{7}$

Observation of this phenomenon has mostly been limited to inorganic crystals, including ammonium dihydrogen phosphate/arsenate, ${ }^{8}$ and chromium oxide hydroxide. ${ }^{9}$ In the organic solid state, historically, there have been few reported instances of isotopic polymorphism, though pyridine is a notable exception. ${ }^{10}$ In the last 30 years, there has been a growing number of reports of isotopic polymorphism in other systems, including: preferred

\footnotetext{
a ISIS Neutron and Muon Facility, Rutherford Appleton Laboratory, Didcot, U.K.; Tel: +44(0)1235 445385; E-mail: nick.funnell@stfc.ac.uk

${ }^{b}$ Diamond Light Source, Diamond House, Rutherford Appleton Laboratory, Didcot, U.K.

${ }^{c}$ The Cambridge Crystallographic Data Centre, 12 Union Road, Cambridge, U.K.

${ }^{d}$ Centre for Science at Extreme Conditions, School of Chemistry, The University of Edinburgh, King's Buildings, West Mains Road, Edinburgh, U.K.; Tel: +44(0)131 6505804; Email: simon.parsons@ed.ac.uk

$\dagger$ Electronic Supplementary Information (ESI) available: CIFs of all structures (CCDC: 2043598-2043607), geometry-optimised structures, calculated vibrational frequencies, crystal structure refinement statistics, low-temperature neutron diffraction experiment details and results.
}

polymorph selection in glycine, ${ }^{11}$ and the ROY family of crystal structures; ${ }^{12}$ suppression of a low-temperature form in pyridine$\mathrm{N}$-oxide; ${ }^{13}$ co-existence of a metastable phase in acetaldehyde; ${ }^{14}$ and determination of the pyridine- $\mathrm{d}_{5}$ crystal structure, ${ }^{15}$ as well as the related acridine molecule. ${ }^{16}$. This behaviour extends to cocrystals, which includes 4-methylpyridine pentachlorophenol, ${ }^{7,17}$ imidazole:terephthalic acid, ${ }^{18}$ and the 'doubly' polymorphic isonicotinamide:oxalic acid. ${ }^{19}$

The first of these co-crystals-4-methylpyridine pentachlorophenol (hereafter 'MPPCP'), shown in Figure 1, was one of the earliest reported instances of organic isotopic polymorphism, first identified through non-identical IR and UV spectra for the two isotopologues. ${ }^{17} \mathrm{X}$-ray structure determination showed that isotopically normal MP and PCP components co-crystallise in the triclinic space group $P \overline{1}$ (Cambridge Structural Database refcode: GADGUN). ${ }^{20}$ The deuterated analogue is observed to crystallise with monoclinic symmetry $C c$ (CSD refcode: GADGUN01) instead.

The difference in crystal structures is accompanied by a change in the interaction between the MP and PCP molecules; in the triclinic structure, they are bridged by a particularly short, directional, hydrogen bond $(\mathrm{H} \cdots \mathrm{N}=1.47(6) \AA)$ and a somewhat longer H-bond interaction in the monoclinic form (1.85(5) $\AA$ ). The shorter of these is a low-barrier H-bond, where neutron diffraction showed that the proton can be shuttled along the bond with varying temperature. ${ }^{21}$ This H-bond is in fact critically important for the differing isotope-induced structural behaviours; ${ }^{1} \mathrm{H}$ MAS NMR investigations showed that the polymorphism is driven exclusively by the proton at the phenolic position - if all the ${ }^{1} \mathrm{H}$ atoms in the structure are substituted, with the exception of the phenolic site, the result is still the $P \overline{1}$ polymorph. ${ }^{7}$ In the same study, attempts were made to encourage growth 
a<smiles>Cc1ccncc1</smiles>

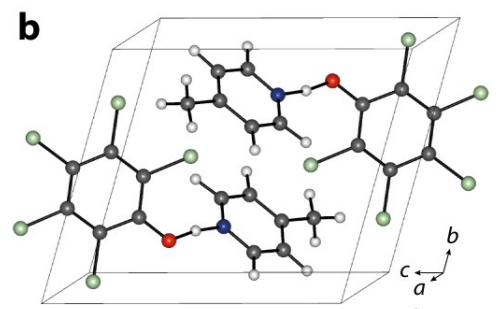

C

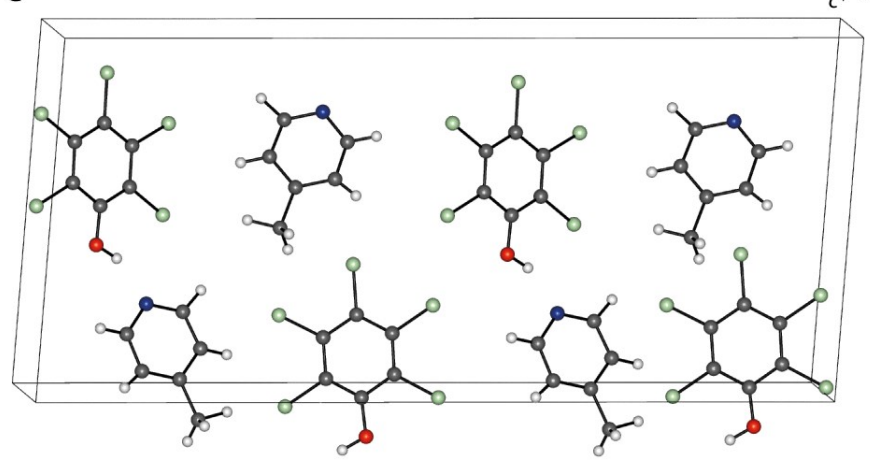

Fig. 1 a) The molecular structures of 4-methylpyridine (MP) and pentachlorophenol (PCP). Unit cell packing in b) triclinic $P \overline{1}$ for the hydrogenated from and $\mathbf{c}$ ) monoclinic $C c$ when the phenolic hydrogen is subtstituted for deuterium. The apparent covalent bond between MP and PCP in b) reflects the very short $\mathrm{N}-\mathrm{H} \cdots \mathrm{O}$ interaction between the two molecules. Carbon atoms are shown in dark grey, oxygen in red, nitrogen in blue, chlorine in green, and hydrogen/deuterium in light grey.

of monoclinic-hydrogenated and triclinic-deuterated crystals by seeding solutions with crystalline samples of the opposite phase. This was reported as being largely unsuccessful; only a small quantity of the monoclinic form was grown using deuterium-free solutions and no triclinic crystals were observed at all for the partially-deuterated samples. Neither could the choice of polymorph be influenced by repeated melting/freezing of the crystal samples.

For brevity, we will refer to monoclinic and triclinic forms as ' $\mathrm{M}$ ' and ' $\mathrm{T}$ ', and suffix these with ' $\mathrm{H}$ ' or ' $\mathrm{D}$ ', to indicate the isotope being used. Thus, the two forms known to exist readily are $\mathrm{TH}$ and MD (and will hereafter appear underlined); MH has only occurred in low concentrations in seeding experiments, and TD has yet to be observed at all. The aim of this investigation is to seek the conditions under which the last two of these polymorphs might be obtained. In particular, the monoclinic crystal structure is more efficiently packed- $355.7 \AA^{3}$ per formula unit, giving a density of $1.683 \mathrm{gcm}^{-3}$ (cf. $363.0 \AA^{3}$ and $1.644 \mathrm{gcm}^{-3}$ for $\mathrm{TH}$ )and so we seek to use pressure to overcome the formation of the shorter H-bond, forcing the growth of the MH structure.

\section{Experimental}

\subsection{Direct compression of a TH single crystal sample}

4-Methylpyridine $(0.18 \mathrm{~mL}, 1.85 \mathrm{mmol})$ and pentachlorophenol $(0.495 \mathrm{~g}, 1.859 \mathrm{mmol})$ were obtained from Sigma-Aldrich and were mixed in a 1:1 molar ratio then refluxed in carbon tetrachloride for 20 minutes. Crystals were grown by slow evaporation of the resulting solution.
A single crystal was loaded in a Merrill-Bassett diamond anvil cell (DAC) with an opening angle of $80^{\circ}$, equipped with Boehler-Almax diamonds, with $600 \mu \mathrm{m}$ culets, a tungsten gasket, and tungsten carbide backing plates. ${ }^{22,23} \mathrm{~A}$ small ruby chip was included as a pressure calibrant, using the ruby fluorescence method to measure the pressure, ${ }^{24}$ and a $1: 1$ volume mixture of pentane:isopentane was loaded as a pressure-transmitting medium. ${ }^{25}$ Diffraction data were then collected at the pressures $0.21,0.57,1.47,2.86,3.75,4.56,4.57$, and $4.59 \mathrm{GPa}$.

Diffraction data for the direct compression of $\mathrm{TH}$ were collected using a Rigaku Saturn 724 CCD detector with siliconmonochromated synchrotron radiation $(\lambda=0.6889 \AA)$ on beamline I19 at the Diamond Light Source. ${ }^{26}$ Frames were recorded with $1 \mathrm{~s}$ exposures with a scan width of $1^{\circ}$, scanning about the $\phi$ and $\omega$ circles. The three highest-pressure datasets (4.564.59 GPa) correspond to three mounting orientations of the DAC; the different pressures are all within error of each other, according to the limitations of the ruby fluorescence measurement. These data were merged into a single dataset to improve completeness. The images were converted from Rigaku to Bruker format using the program ECLIPSE. ${ }^{27}$ Data were integrated using SAINT and absorption corrections were carried out with the program SADABS. ${ }^{28,29}$

\subsection{In situ crystal growth of $\mathrm{MH}$ at high pressure}

4-methylpyridine $(0.23 \mathrm{~mL}, 2.36 \mathrm{mmol})$ and pentachlorophenol $(0.6400 \mathrm{~g}, 2.403 \mathrm{mmol})$ were dissolved in a minimum volume of acetonitrile. The solution was loaded into a DAC set up as described above. Crystallisation was induced by increasing pressure to $0.5 \mathrm{GPa}$. The majority of the crystallites formed were melted with a hot-air gun until very few seeds remained. These were allowed to grow as the cell cooled to room temperature. Diffraction data were then collected; the average pressure in the cell during the data collection was measured to be $0.4 \mathrm{GPa}$. The acetonitrile solvent also crystallised at this pressure.

Data for the MH crystal grown at pressure were also collected on I19 $(\lambda=0.5159 \AA)$, using the same collection strategy as for $\underline{\mathrm{TH}}$. Four domains of MH were identified and each were integrated separately. One domain was discarded owing to poor integration statistics. The three remaining domains were merged in SORTAV, ${ }^{30}$ increasing data completeness (individual: 39/42/49\% to $0.92 \AA$; merged: $63 \%$ to $0.92 \AA$ ).

\subsection{High-pressure crystal structure refinement}

Structure refinements against $F^{2}$ were carried out in Crystals using the starting coordinates for TH (GADGUN) and MD (GADGUN01) determined at ambient pressure. ${ }^{17}$ Due to the low completeness of the datasets (ranging between $20.8-29.8 \%$ to 0.76 $0.90 \AA$ for $\underline{\mathrm{TH}}$ and $63.4 \%$ to $0.92 \AA$ for $\mathrm{MH}$ ), all primary bond distances and angles were restrained to values informed by the starting models. In the TH sample, all non-hydrogen atoms were refined isotropically, whereas anisotropic refinement was possible for the relatively electron-heavy chlorine atoms in $\mathrm{MH}$.

Hydrogen atoms were all placed geometrically, except for those on the methyl group in $\mathrm{MH}$, which were located in a difference 
map. All their geometries were regularised with restraints. The phenolic hydrogen atom could not be located from the diffraction data and was included in the same position as observed in the ambient-pressure structure. All hydrogen atoms were subsequently constrained to ride on their host atoms.

\subsection{Recovery of MH to ambient pressure}

An MH crystal, grown at high pressure, was recovered from the DAC to ambient conditions. As pressure was decreased, the solubility of the crystal in the acetonitrile solvent increased, reducing its size. Cooling the DAC with dry ice prevented complete dissolution of the crystal, which measured $0.05 \times 0.07 \times 0.17 \mathrm{~mm}$ on recovery.

Diffraction data for the recovered crystal were collected on an Agilent Technologies SuperNova, with an Atlas detector, at 150 and $80 \mathrm{~K}$ using $\mathrm{Cu} K_{\alpha}$ radiation. Low-angle reflections were collected with $3 \mathrm{~s}$ exposures and high-angle reflections, $30 \mathrm{~s}$, scanning about the $\omega$ circle with frame widths of $1^{\circ}$. Data were integrated and corrected for absorption using CrysAlisPro. ${ }^{31}$ Data were merged in SORTAV. Structure refinements against $F^{2}$ were carried out in Crystals. All atoms were located in a difference map and all non-H atoms were refined anisotropically. The geometries of the $\mathrm{H}$ atoms were regularised with restraints and were subsequently constrained to ride on their host atoms.

Crystal and refinement statistics for all MH structures and select $\mathrm{TH}$ structures are listed in Table 1. Complete statistics for all datasets are available in the ESI.

\subsection{Vibrational frequency calculations}

DFT calculations were performed using the DMOL package implemented in the Materials Studio software. ${ }^{32}$ The PBE exchangecorrelation functional was used with the Grimme dispersion correction. ${ }^{33,34}$ A numerical basis set (DNP 3.5) was used and Brillouin zone integrations were performed on a $k$-point grid with $0.07 \AA$ spacing. ${ }^{35}$ The total energy convergence was $1 \times 10^{-5} \mathrm{Ha}$ and the maximum displacement allowed was $5 \times 10^{-3} \AA$.

The starting coordinates for the calculations were taken from GADGUN04 for the TH polymorph and the refined structure of the crystal recovered from pressure for the $\mathrm{MH}$ phase; the $\mathrm{MH}$ cell was expressed in its primitive setting. The cell dimensions were initially held fixed whilst the atomic coordinates were allowed to refine. The structures were allowed to relax in $P 1$ prior to the frequency calculations. A second set of frequency calculations were carried out with the cell dimensions being allowed to optimise. The frequencies for TD and MD were obtained by repeating the final frequency calculations for fixed and refined-cell optimisations, but substituting $\mathrm{H}$ with D. For clarity, only the results from the optimisations using fixed cell dimensions are reported here as similar results are obtained when the cell dimensions are allowed to optimise.

\section{Results}

\subsection{Hydrostatic compression of TH}

At ambient pressure and temperature the MD form is more efficiently packed than $\underline{\mathrm{TH}}-355.7$ versus $363.0 \AA^{3}$ per formula unit, respectively, thus it was initially thought possible that pressure would drive a $\underline{\mathrm{TH}} \rightarrow \mathrm{MH}$ transformation via volume reduction. However, when $\underline{\mathrm{TH}}$ was studied by single crystal diffraction up to $4.59 \mathrm{GPa}$, the crystal remained in a compressed form of the triclinic phase. Increasing the pressure above $4.59 \mathrm{GPa}$ led to disintegration of the crystal. Analysis of the principal axes of the strain tensor reveals that one of these directions $(-0.9 \mathbf{a}-0.1 \mathbf{b}-0.3 \mathbf{c})$ is extremely incompressible compared with the other two directions; 1.6(6) $\mathrm{TPa}^{-1}$ versus 22(4) and 19(4) $\mathrm{TPa}^{-1}$-see Figure $2 .{ }^{36}$ This direction is closely aligned with the orientation of the intermolecular hydrogen bond, which straightforwardly accounts for the lack of compressibility along this axis. The trend in sample compression is not continuous, which led to difficulties in fitting a bulk modulus, and the relatively large uncertainties quoted here for axis compressibility. We note that we use the cell dimensions of GADGUN as an input for the ambient pressure structure so as to avoid uncertainty associated with the pressure measurement of our lowest pressure structure inside the DAC; i.e. whether it is truly ambient pressure. Pressure-principal axis plots, and an indicatrix visualisation of the compressibilites are shown in Figure 2.

The MPPCP dimer in the TH structure is notable for its particularly short, centred, intermolecular hydrogen bond, where the proton migrates between the MP and PCP components with temperature. ${ }^{21,37}$ Thus the $\underline{\mathrm{TH}}$ form exists on a thermally-dependent co-crystal-salt continuum. While we cannot locate the phenolic proton from our diffraction data (it was placed geometrically in the refinements), we can comment on the $\mathrm{O} \cdots \mathrm{N}$ distance. At room temperature it measures 2.550 (6) $\AA$, and shortens with decreasing temperature, reducing to $2.506(2) \AA$ at $20 \mathrm{~K} .{ }^{37}$ At lower temperatures, the proton is more closely located to the nitrogen atom. At the highest pressure measured here, we find the $\mathrm{O} \cdots \mathrm{N}$ separation is $2.42(3) \AA$-shorter than at $20 \mathrm{~K}$, though the difference is marginally below a $3 \sigma$ level of significance $(2.9 \sigma)$. It would seem reasonable to suggest that at high pressures, the proton is more likely located closer to the nitrogen atom, given the trend with decreasing temperature.

In organic materials, volume minimisation is often achieved via reduction in void space in the unit cell. We calculate the volume of both the voids and the network of intermolecular contactsthe space enclosed within the VdW radii of all atoms-using a Monte-Carlo procedure, as described in Ref. 38. These are plotted in Figure 2. As pressure is increased, there is no indication that the void volume is reaching a lower limit, i.e. it does not approach a constant value. However, the network volume presents a different picture, where it decreases abruptly above $3.75 \mathrm{GPa}$. In L-histidine, this same analysis showed that volume discontinuities in the molecular network preceded phase transitions, ${ }^{38}$ which suggests that the $\underline{\mathrm{TH}}$ sample may also be attempting to undergo a phase transition when it disintegrates.

Details of the crystal structure refinement at $4.59 \mathrm{GPa}$ are given 


\begin{tabular}{|c|c|c|c|c|c|c|}
\hline Pressure/temperature & $0.21 \mathrm{GPa}$ & $2.86 \mathrm{GPa}$ & $4.57 \mathrm{GPa}$ & $0.4 \mathrm{GPa}$ & $150 \mathrm{~K}$ & $80 \mathrm{~K}$ \\
\hline X-ray wavelength / $\AA$ & 0.6889 & 0.6889 & 0.6889 & 0.5159 & 1.5406 & 1.5406 \\
\hline Chemical formula & $\mathrm{C}_{10} \mathrm{H}_{8} \mathrm{~N}_{1} \mathrm{O}_{1} \mathrm{Cl}_{5}$ & $\mathrm{C}_{10} \mathrm{H}_{8} \mathrm{~N}_{1} \mathrm{O}_{1} \mathrm{Cl}_{5}$ & $\mathrm{C}_{10} \mathrm{H}_{8} \mathrm{~N}_{1} \mathrm{O}_{1} \mathrm{Cl}_{5}$ & $\mathrm{C}_{10} \mathrm{H}_{8} \mathrm{~N}_{1} \mathrm{O}_{1} \mathrm{Cl}_{5}$ & $\mathrm{C}_{10} \mathrm{H}_{8} \mathrm{~N}_{1} \mathrm{O}_{1} \mathrm{Cl}_{5}$ & $\mathrm{C}_{10} \mathrm{H}_{8} \mathrm{~N}_{1} \mathrm{O}_{1} \mathrm{Cl}_{5}$ \\
\hline Formula weight $/ \mathrm{g} \mathrm{mol}^{-1}$ & 335.44 & 335.44 & 335.44 & 335.44 & 335.44 & 335.44 \\
\hline Crystal system & Triclinic & Triclinic & Triclinic & Monoclinic & Monoclinic & Monoclinic \\
\hline Space group & $P \overline{1}$ & $P \overline{1}$ & $P \overline{1}$ & $C c$ & $C c$ & $C c$ \\
\hline$a$-axis / 丹 & $7.39220(3)$ & $7.09540(3)$ & $7.02900(3)$ & $3.8385(8)$ & $3.86184(17)$ & $3.8299(3)$ \\
\hline$b$-axis / $\AA$ & $8.92720(8)$ & $8.23750(7)$ & $8.00850(6)$ & $27.440(6)$ & $27.5870(14)$ & $27.5220(19)$ \\
\hline$c$-axis / $\AA$ & $13.65240(9)$ & $12.39660(6)$ & $12.08050(6)$ & $12.924(5)$ & $13.0065(5)$ & $13.0115(16)$ \\
\hline$\alpha /^{\circ}$ & $100.317(14)$ & $100.861(11)$ & $101.110(10)$ & 90.0 & 90.0 & 90.0 \\
\hline$\beta /{ }^{\circ}$ & $118.354(9)$ & $114.958(7)$ & $114.232(7)$ & $95.52(3)$ & $95.678(4)$ & $95.766(10)$ \\
\hline$\gamma /{ }^{\circ}$ & 103.641(9) & $104.500(8)$ & $104.203(8)$ & 90.0 & 90.0 & 90.0 \\
\hline Volume / $\AA^{3}$ & $724.49(12)$ & $599.22(8)$ & $567.09(7)$ & $1355.0(5)$ & $1378.87(11)$ & $1364.6(2)$ \\
\hline Density / $\mathrm{gcm}^{-3}$ & 1.648 & 1.992 & 2.105 & 1.762 & 1.731 & 1.750 \\
\hline Parameters & 77 & 77 & 77 & 102 & 173 & 275 \\
\hline No. independent reflections & 620 & 548 & 669 & 1062 & 2082 & 2035 \\
\hline$R 1, w R(I>2 \sigma)$ & $0.139,0.389$ & $0.118,0.385$ & $0.135,0.424$ & $0.062,0.124$ & $0.048,0.121$ & $0.060,0.153$ \\
\hline Goodness of fit & 1.7140 & 1.8296 & 1.9834 & 0.9871 & 0.9623 & 1.0078 \\
\hline$\mu / \mathrm{cm}^{-1}$ & 0.893 & 1.080 & 1.141 & 0.423 & 9.504 & 9.603 \\
\hline$\Delta \rho_{\max }, \Delta \rho_{\min } / \mathrm{e} \AA^{-3}$ & $-0.55,0.63$ & $-0.64,0.74$ & $-1.11,1.07$ & $-1.21,1.02$ & $-0.39,0.73$ & $-0.33,0.49$ \\
\hline Completeness, resolution / $\AA$ & $29.76 \%, 0.90$ & $22.84 \%, 0.80$ & $24.48 \%, 0.76$ & $63.44 \%, 0.92$ & $97.77 \%, 0.81$ & $96.54 \%, 0.80$ \\
\hline
\end{tabular}

in Table 1; details for all pressure points, and CIFs of each, are available in the ESI.

\subsection{Pressure-induced growth of $\mathrm{MH}$}

Following the observation that a transition to a monoclinic form could not be driven by direct compression of $\underline{\mathrm{TH}}$, we instead used pressure to induce crystallisation from a supersaturated acetonitrile solution. This approach has been used with success to bypass kinetic barriers that otherwise prevent molecules reorganising into a thermodynamically-stable structure. ${ }^{39,40}$ Crystallisation was induced at $0.5 \mathrm{GPa}$ and via careful thermal cycling, we were able to reduce the number of crystallites, such that only four sample domains could be identifed. These were all indexed on the $C c$ cell $a=3.8385(6), b=27.440(3), c=12.924(3)$, and $\beta=95.52(3)^{\circ}$, where full refinement showed the structure to be the same as the MD form.

Using a different set of pressure-grown MH crystals, we found that they could be recovered from the DAC to ambient pressure, under the conditions noted in the Experimental section, and did not transform to the $\underline{\mathrm{TH}}$ polymorph. Complete diffraction data were collected in-house at 150 and $80 \mathrm{~K}-\mathrm{CIFs}$ are available in the ESI. Images of the crystal in the DAC sample chamber, and the recovered crystal mounted on a goniometer head, are shown in Figure 3. The low-temperature behaviour of the $\mathrm{MH}$ form follows that of MD; the asymmetric unit also becomes disordered over two positions with uneven occupancies. ${ }^{41}$ There is an indication of cell doubling in the raw diffraction data at $80 \mathrm{~K}$ although we were unable to obtain a satisfactory refinement in $P c$. The partial occupancies in the $C c$ cell are 0.774 and 0.226 , suggesting that if there is a transition to a larger cell, it is incomplete. a

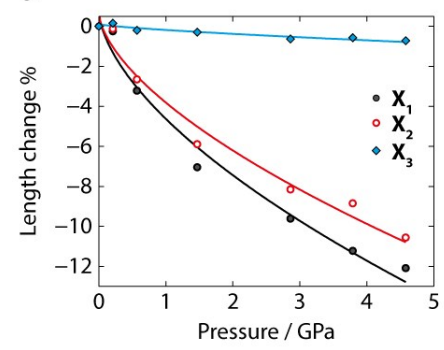

C

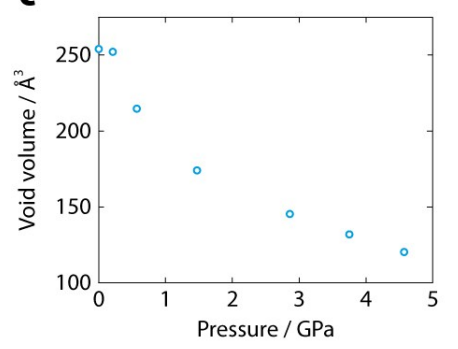

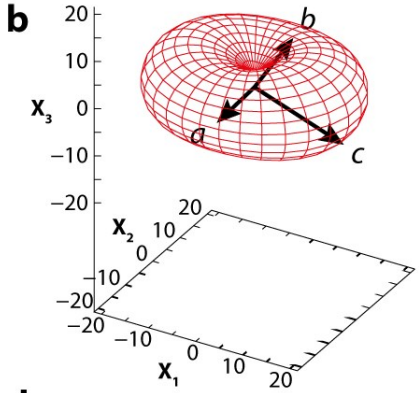

d

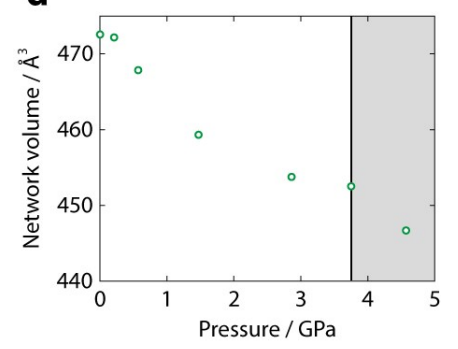

Fig. 2 a) Percentage change in length of $\underline{\mathrm{TH}}$ principal axes. $\mathbf{X}_{1}=-0.36 \mathbf{a}+0.20 \mathbf{b}+0.91 \mathbf{c} ; \mathbf{X}_{2}=0.30 \mathbf{a}+0.95 \mathbf{b}+0.10 \mathbf{c}$;

$\mathbf{X}_{3}=-0.93 \mathbf{a}-0.12 \mathbf{b}-0.34 \mathbf{c}$. Non-continuous trends in axis compression leads to difficulties in obtaining good fits. The fit here is an empirical function used by the PASCal program. ${ }^{36}$ b) The axis compressibility trends still provide a clear indication of the most and least compressible directions, visualised as an indicatrix, shown relative to the triclinic cell axes. Plot axes are principal axis compressibilities, with units $\mathrm{TPa}^{-1}$. Volume of $\mathbf{c}$ ) interstitial voids and d) the 'molecular network'. An abrupt decrease in the latter is indicated by the shaded region. 


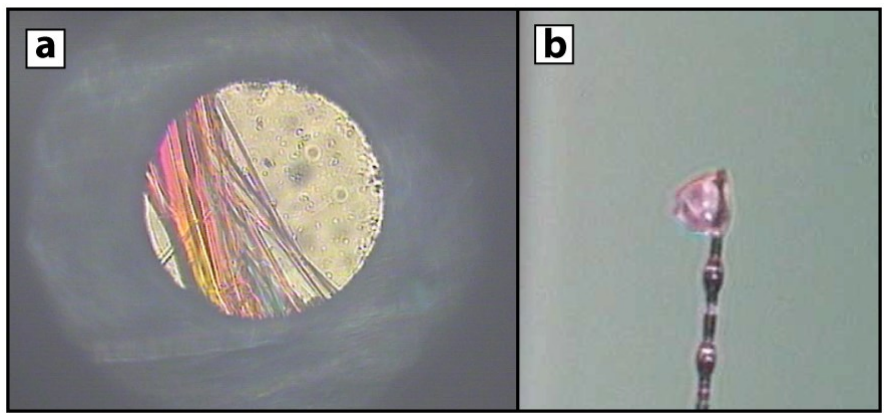

Fig. 3 a) Multiple MH crystals, shown within the DAC sample chamber. Colours in the image are generated by microscope polarisation. b) A recovered $\mathrm{MH}$ crystal mounted on a goniometer head fibre. The crystal is colourless; the pink colour here is due to diffractometer cabinet lighting.

\section{Discussion}

\subsection{Crystal engineering with pressure}

The $\mathrm{MH}$ form was acquired directly from solution, thus avoiding the kinetic barriers which trap the TH structure-it is likely the thermodynamically stable product when crystallisation is induced at $0.5 \mathrm{GPa}$. Through direct comparison of formula-unit volumes, we are able to quantify the change in packing energy $P \Delta V$, one of the components of Gibbs free energy $G=U+P V-T S$. At $0.4 \mathrm{GPa}$, the formula-unit volume of $\mathrm{MH}$ is $338.75 \AA^{3}$. An approximate fit to the $\underline{\mathrm{TH}}$ pressure-volume data finds the formula-unit volume at $0.4 \mathrm{GPa}$ is $348.15 \AA^{3}$-a difference of $9.4 \AA^{3}$ between the two forms. This improved packing in MH leads to a $2.26 \mathrm{kJmol}^{-1}$ energy reward in $P V$, which is greater than the energy difference typically seen between polymorphs in the organic solid state; often $2 \mathrm{kJmol}^{-1}$ or less. ${ }^{42}$ Though the $U$ and $T S$ terms will also influence the difference in polymorph energies, the fact that crystallisation of $\mathrm{MH}$ does not occur without applying pressure suggests that volume reduction is crucial for its formation.

Once recovered from the DAC, the efficiently-packed MH polymorph exhibited stability to ambient pressure between room temperature and $80 \mathrm{~K}$. Previously the MH phase has only ever been observed in very small quantities when seeding the liquid protonated compound with $\mathrm{MD}$ crystals, but the exclusive formation of MH in the DAC reflects the need to pack efficiently at elevated pressure. Furthermore, this highlights pressure as a crystal engineering tool; allowing the user to access crystal forms which, although stable under ambient conditions, are rendered unobtainable by kinetics. Using pressure in this way is not new, ${ }^{39,40}$ but this is one of very few examples of using pressure to suppress polymorphism induced by isotopic substitution in the organic solid state. ${ }^{15}$

\subsection{Relative polymorph stabilities}

The origin of isotopic polymorphism in MPPCP is discussed in Ref. 43, where the authors proposed that the difference in vibrational zero point energies (ZPE) of the respective hydrogen/deuterium bonds might be highly influential in determining the crystal structure. We redraw the hydrogen-bond potential schematics, out-

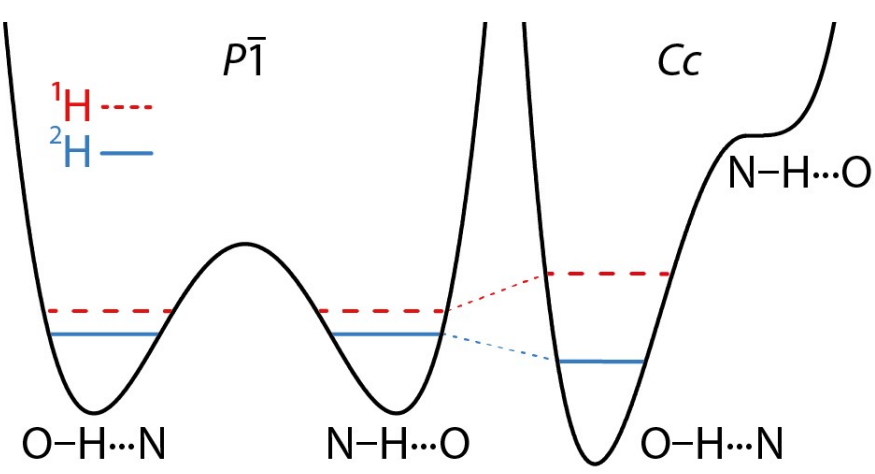

Fig. 4 Zero point energy schematic for hydrogen/deuterium-bonds, proposed in Ref. 43 for the $P \overline{1}$ (left) and $C c$ (right) forms. The minima in each potential correspond to hydrogen location toward either oxygen or nitrogen atoms. Hydrogen ZPEs are indicated by red, dashed lines, and deuterium by blue, solid lines.

lined in this study, in Figure 4. For $P \overline{1}$ the potential is symmetric and has a low barrier to allow for proton migration between the MP and PCP molecules, and $C c$ is a single asymmetric energy well, where the separation between H/D ZPEs is greater, such that they 'straddle' the ZPEs of the triclinic alternative. This implies the stability series $\underline{\mathrm{MD}}>\mathrm{TD}>\underline{\mathrm{TH}}>\mathrm{MH}$.

We have quantified the relative crystal structure stabilities, at ambient pressure, using periodic DFT. The ZPEs we calculate are derived from all $\Gamma$-point vibrations, not just those of the hydrogen bond. In addition to calculating each ZPE, we have also determined the internal energy $U_{0}$, and statistical estimates of vibrational enthalpy $H_{\text {vib }}$ and the temperature $\times$ entropy $T S$ terms. Here, the effects of temperature can also be modelled, within a harmonic approximation. The $P V$ term can be omitted as $P \approx 0$; thus the direct energy advantage conferred by the density of the monoclinic structure is greatly reduced. However, the spatial separation of molecules also has an impact on long-range phonon modes, which in turn affect $H_{\text {vib }}$ and $T S$.

The importance of considering the role of entropy in stabilising crystal structures is well-recognised. A new polymorph of salicylamide was crystallised at high pressure, where its cohesive energy $U_{0}$ was reduced relative to that of the ambient-pressure form, but was ultimately compensated for by entropy. ${ }^{44}$ This was thought to arise from elongated hydrogen bonds, which resulted in lower phonon frequencies and therefore higher entropy. The same behaviour has also been seen in biurea where a low-pressure form was stabilised via entropic contributions, before eventually being overcome by more favourable internal energy and packing in a closely-related conformational polymorph. ${ }^{45}$ An assessment of a large range ( 475 pairs) of crystalline polymorphs indicated that vibrational energy contributions resulted in diverging free energy curves versus temperature for $38 \%$ of the structures investigated. ${ }^{46}$ Thus, ranking relative polymorph stabilities on lattice energy alone can often be too simplistic.

DFT calculations were carried out as detailed in the Experimental section. The level of agreement between the observed and optimised crystal structures of $\underline{\mathrm{TH}}$ and $\underline{\mathrm{MD}}$ was excellent, with root mean square deviations of 0.081 and 0.072 , respectively, calculated for a cluster of 15 molecules. ${ }^{47}$ The optimised 
TH structure showed a preference to locate the proton closer to the nitrogen atom, reflecting the behaviour seen experimentally at low temperature-notable as the DFT calculation is effectively performed at $0 \mathrm{~K}$. Atomic coordinates of the optimised structures, following relaxation in $P 1$, are available in the ESI in .res format. Phonon frequencies calculated for $\mathrm{TH}, \mathrm{TD}, \mathrm{MH}$, and $\mathrm{MD}$, were used to evaluate ZPEs and the thermal contributions to enthalpy and the entropy at temperatures between 298 and $700 \mathrm{~K}$. The procedure used followed that described by Rivera $e t$ al, ${ }^{48}$ yielding Gibbs free energies of the polymorphs as a function of temperature. These are calculated as $E_{\mathrm{DFT}+\mathrm{D}}+\mathrm{ZPE}+H_{\mathrm{vib}}-T S$, and are plotted in Figure 5, in which energies are quoted relative to that of the TH polymorph. Calculations have been performed up to $700 \mathrm{~K}$ in order to clearly illustrate the trend for each form; the absolute temperature values are unimportant. We note that the crystal structure of $\underline{\mathrm{TH}}$ is known to melt at ca. $350 \mathrm{~K} .{ }^{37}$

The plot shows that the polymorph hierarchy changes with temperature as the gradients between the monoclinc and triclinic forms are very different. The order proposed in Ref. 43 occurs within a relatively narrow temperature range of $392-405 \mathrm{~K}$. Below $392 \mathrm{~K}$, the stability order is $\mathrm{TD}>\underline{\mathrm{MD}}>\mathrm{TH}>\mathrm{MH}$, and above $400 \mathrm{~K}$ it is $\underline{\mathrm{MD}}>\mathrm{TD}>\mathrm{MH}>\underline{\mathrm{TH}}$. At all temperatures, the heavier, deuterated, structures are more stable than their hydrogenated counterparts. When the phonon calculations are repeated using optimised cell dimensions, then the order in Ref. 43 is shifted to ca. $360-375 \mathrm{~K}$.

The difference between the stability series, proposed on the basis of room temperature observations, and that calculated here, can probably be ascribed to the approximations employed in calculating the vibration frequencies; our calculations are performed at the $\Gamma$-point of the Brillouin zone. Notably, we assume harmonic behaviour and neglect contributions from acoustic modes away from the zone centre, as well as the effects of phonon dispersion. However, the general trend in relative free energies is clear. The difference in gradient between each polymorph originates from either, or both of, the $H_{\mathrm{vib}}$ and $T S$ terms, as these are temperature dependent. The largest contribution to these terms come from low-frequency vibrations; Table 2 shows the calculated $H_{\text {vib }}$ and $T S$ terms at 298.15 and $700 \mathrm{~K}$, corresponding to the regions 0-250, 250-500 and $>500 \mathrm{~cm}^{-1}$ for each polymorph. The Table shows that although the $H_{\text {vib }}$ term certainly becomes more influential as temperature is increased, the $T S$ term is mostly responsible for determining the gradients of the free energy versus temperature plots. The low-frequency vibrations at $0-250 \mathrm{~cm}^{-1}$ are evidently the main contributors to these terms-the $>500 \mathrm{~cm}^{-1}$ region spans a wider frequency range than the other entries in Table 2 combined, but these vibrations influence the free energies to a far lesser extent. The calculated vibrational frequencies are made available in a supplementary .csv document.

\subsection{Proposed experimental conditions for growth of TD}

The TD polymorph has yet to be observed experimentally. Our frequency calculations suggest that this form should become energetically favoured at lower temperatures-presumably at least 60 degrees below room temperature, given the discrepancy of
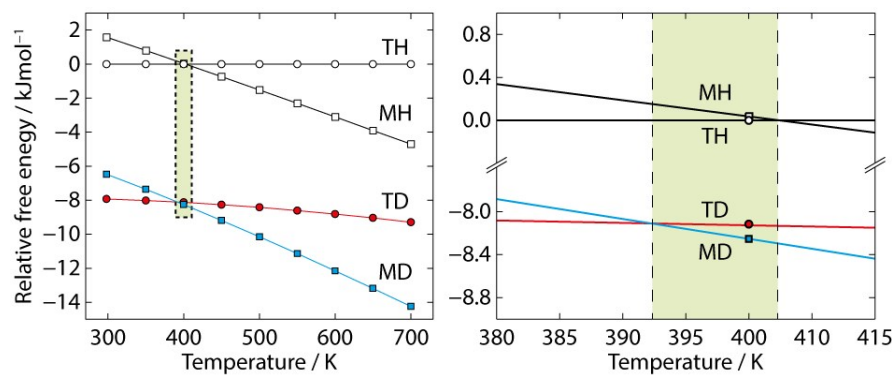

Fig. 5 Calculated Gibbs free energies, shown relative to the $\underline{\mathrm{TH}}$ polymorph. $\mathrm{TH}$ is shown by open circles, MH by open squares, TD by filled red circles, and MD by filled blue squares. The narrow range over which the stability series observed at ambient conditions is indicated by the shaded green rectangle; this region is expanded, and plotted, on the right hand side.

that same magnitude between our calculations (optimised cell) and experimental observation.

To this end, we carried out a neutron investigation on a perdeuterated sample (i.e. MPPCP- $\mathrm{d}_{8}$ ) using the POLARIS instrument at the ISIS Neutron and Muon Facility, ${ }^{49}$ aiming to take advantage of the H/D discriminating ability of neutrons. In all measurements, the sample was dissolved in perdeuterated acetonitrile and the solution was cooled to induce crystallisation. Measurements were made between temperatures 183-250 K, using solutions of varying concentrations, but in all cases the $\underline{\mathrm{MD}}$ form resulted. It appeared we were able to promote formation of an amorphous form, indicated by very broad, but structured, features, which again on slight warming, crystallised in $C c$. One diffraction pattern, available in the ESI, showed a very weak, short-lived feature, that was inconsistent with a $C c$ crystal structure, but could be accounted for with the triclinic cell. However, nothing conclusive was identified.

Accessing the thermodynamically stable TD form at low temperature may be confounded by the readiness with which $\mathrm{MD}$ forms on cooling. When the sample is crash-cooled by, e.g. immersion in liquid nitrogen, another difficulty is presented in removal of the acetonitrile solvent at these very low temperatures, in order to encourage crystallisation. Obtaining the TD polymorph remains an open challenge for the crystal engineering community, but we hope that our calculations and measurements here provide a guide for the conditions that are likely to be required for growth of this form.

\section{Conclusions}

We have used pressure to suppress the effects of isotopic polymorphism in an organic system, and recover a crystal form that is stable, but otherwise unobtainable, at ambient conditions. The $\mathrm{MH}$ form we have grown has otherwise only been obtained in small quantities after cross-seeding with crystals of its deuterated isotopologue. At $0.4 \mathrm{GPa}$, on the basis of unit cell volumes, the energetic advantage in $P \Delta V$ to pack with $C c$ symmetry in preference to $P \overline{1}$ is $2.26 \mathrm{kJmol}^{-1}$ per formula unit which, evidently, is sufficient to make $\mathrm{MH}$ energetically preferred to the $\underline{\mathrm{TH}}$ form. Thus the narrow energy difference between all the MPPCP forms 
Table 2 Temperature-dependent, vibrational contributions to Gibbs free energy for each form. All energies are given in $\mathrm{kJmol}^{-1}$. Energy contributions are divided between frequency regions, showing the larger contributions at a given temperature come from lower-frequency vibrations. Note that the $>500 \mathrm{~cm}^{-1}$ entry encompasses a much larger frequency range than the other columns.

\begin{tabular}{ccccccc}
\hline \multirow{2}{*}{ Polymorph } & \multicolumn{2}{c}{$\mathbf{0 - 2 5 0} \mathbf{c m}^{-1}$} & \multicolumn{2}{c}{$\mathbf{2 5 0 - 5 0 0} \mathbf{c m}^{-1}$} & \multicolumn{2}{c}{$>\mathbf{5 0 0} \mathbf{c m}^{-1}$} \\
& $\mathbf{2 9 8 . 1 5 ~ K}$ & $\mathbf{7 0 0 . 0 K}$ & $\mathbf{2 9 8 . 1 5} \mathbf{K}$ & $\mathbf{7 0 0 . 0 K}$ & $\mathbf{2 9 8 . 1 5} \mathbf{K}$ & $\mathbf{7 0 0 . 0 K}$ \\
\hline TH $H_{\text {vib }}$ & 36.67 & 104.00 & 8.80 & 38.54 & 5.19 & 69.30 \\
MH $H_{\text {vib }}$ & 36.03 & 100.23 & 9.75 & 41.30 & 5.52 & 71.44 \\
TD $H_{\text {vib }}$ & 36.70 & 104.03 & 8.81 & 38.56 & 5.42 & 71.33 \\
MD $H_{\text {vib }}$ & 36.68 & 102.46 & 9.45 & 40.82 & 5.53 & 71.66 \\
\hline$\underline{\text { TH } T S}$ & 81.19 & 290.45 & 13.34 & 74.89 & 6.63 & 103.74 \\
MH $T S$ & 83.57 & 291.41 & 15.01 & 81.52 & 7.06 & 107.40 \\
TD $T S$ & 81.28 & 290.66 & 13.36 & 74.94 & 6.93 & 107.05 \\
MD $T S$ & 84.70 & 296.41 & 14.42 & 79.84 & 7.06 & 107.72 \\
\hline
\end{tabular}

proposed in the ZPE model of Ref. 43 fits the experimental observation that we make here. The predicted hierarchy of polymorph stabilities has been reproduced by periodic DFT and statistical thermodynamics calculations, albeit over a narrow temperature range, at ca. $100 \mathrm{~K}$ higher than observed experimentally. We have identified that the TD form is likely to become thermodynamically favoured at low temperatures, but have been unable to observe conclusive growth of this form due to the difficulty in reaching these conditions without first forming $\underline{\mathrm{TH}}$, where the sample remains kinetically trapped.

\section{Conflicts of interest}

There are no conflicts to declare.

\section{Acknowledgements}

We thank EPSRC and CCDC for studentship funding and STFC for the provision of neutron and synchrotron beamtime.

\section{References}

1 S. J. Fisher and J. R. Helliwell, Acta Crystallogr., 2008, A64, 359-367.

2 K. Merz and A. Kupka, Cryst. Growth Des., 2015, 15, 15531558.

3 W. Press, J. Eckert, D. E. Cox, C. Rotter and W. Kamitakahara, Phys. Rev. B, 1976, 14, 1983-1988.

4 M. I. McMahon, R. J. Nelmes, W. F. Kuhs, R. Dorwarth, R. O. Piltz and Z. Tun, Nature, 1990, 348, 317-319.

5 S. Horiuchi, R. Kumai and Y. Tokura, J. Am. Chem. Soc., 2005, 127, 5010-5011.

6 T. Jin and W. Zhang, CrystEngComm, 2019, 21, 4238-4242.

7 J. Zhou, Y.-S. Kye and G. S. Harbison, J. Chem. Am. Soc., 2004, 126, 8392-8393.

8 A. A. Khan and H. Baur, Werner, Acta Crystallogr., 1973, B29, 2721-2726.

9 T. Fujihara, M. Ichikawa, T. Gustafsson, I. Olovsson and T. Tsuchida, J. Phys. Chem. Solids, 2002, 63, 309-315.

10 E. Castellucci, G. Sbrana and F. D. Verderame, J. Chem. Phys., 1969, 51, 3762-3770.

11 C. E. Hughes and K. D. M. Harris, New J. Chem., 2009, 33, 713-716.
12 J. Falk, D. Hofmann and K. Merz, IUCrJ, 2018, 5, 569-573.

13 V. Vasylyeva, T. Kedziorski, N. Metzler-Nolte, C. Schauerte and K. Merz, Cryst. Growth Des., 2010, 10, 4224-4226.

14 R. M. Ibberson, O. Yamamuro and T. Matsuo, J. Mol. Struct., 2000, 520, 265-272.

15 S. Crawford, M. T. Kirchner, D. Bläser, R. Boese, W. I. F. David, A. Dawson, A. Gehrke, R. M. Ibberson, W. G. Marshall, S. Parsons and O. Yamamuro, Angew. Chem. Int. Ed., 2009, 48, 755757.

16 A. Kupka, V. Vasylyeva, D. W. M. Hofmann, K. V. Yusenko and K. Merz, Cryst. Growth Des., 2012, 12, 5966-5971.

17 I. Majerz, Z. Malarski and T. Lis, J. Mol. Struct., 1990, 240, 47-58.

18 C. Shi, X. Zhang, C.-H. Yu, Y.-F. Yao and W. Zhang, Nat. Commun., 2018, 9, 481.

19 M. Schmidtmann, D. S. Middlemiss and C. C. Wilson, CrytEngComm, 2015, 17, 5273-5279.

20 C. R. Groom, I. J. Bruno, M. P. Lightfoot and S. C. Ward, Acta Crystallogr., 2016, B72, 171-179.

21 T. Steiner, I. Majerz and C. C. Wilson, Angew. Chem. Int. Ed., 2001, 40, 2651-2653.

22 L. Merrill and W. A. Bassett, Rev. Sci. Instrum., 1974, 45, 290 294.

23 S. A. Moggach, D. R. Allan, S. Parsons and J. E. Warren, J. Appl. Crystallogr., 2008, 41, 249-251.

24 G. J. Piermarini, S. Block, J. D. Barnett and R. A. Forman, J. Appl. Phys., 1975, 46, 2774-2780.

25 S. Klotz, J.-C. Chervin, P. Munsch and G. L. Marchand, J. Phys. D: Appl. Phys., 2009, 42, 075413.

26 H. Nowell, S. A. Barnett, K. E. Christensen, S. J. Teat and D. R. Allan, J. Synchrotron Rad., 2012, 19, 435-441.

27 S. Parsons, ECLIPSE, 2009.

28 Bruker-Nonius, SAINT version 7, Program for integration of area detector data, 2006.

29 G. Sheldrick, SADABS, 2008.

30 R. H. Blessing, J. Appl. Crystallogr., 1997, 30, 421-426.

31 Agilent, CrysAlis PRO, 2010.

32 B. Delley, J. Chem. Phys., 2000, 113, 7756-7764.

33 J. P. Perdew, K. Burke and M. Ernzerhof, Phys. Rev. Lett., 1996, 77, 3865-3868. 
34 S. Grimme, J. Comput. Chem., 2006, 27, 1787-1799.

35 H. J. Monkhorst and J. D. Pack, Phys. Rev. B-Solid St., 1976, 13(12), 5188-5192.

36 M. J. Cliffe and A. L. Goodwin, J. Appl. Crystallogr., 2012, 45, 1321-1329.

37 L. Rajput, M. Banik, J. R. Yarava, S. Joseph, M. K. Pandey, Y. Nishiyama and G. R. Desiraju, IUCrJ, 2017, 4(4), 20522525.

38 G. Novelli, H. E. Maynard-Casely, G. J. McIntyre, M. R. Warren and S. Parsons, Cryst. Growth. Des., in press.

39 F. P. A. Fabbiani, D. R. Allan, W. I. F. David, S. A. Moggach, S. Parsons and C. R. Pulham, CrystEngComm, 2004, 6, 505511.

40 I. B. Hutchison, A. Delori, X. Wang, K. V. Kamenev, A. J. Urquhart and I. D. H. Oswald, CrystEngComm, 2015, 17, 1778-1782.

41 Z. Malarski, I. Majerz and T. Lis, J. Mol. Struct., 1996, 380, 249-256.

42 C. A. Hunter and R. Prohens, CrystEngComm, 2017, 19, 2326.
43 J. Zhou, Y.-S. Kye, A. I. Kolesnikov and G. S. Harbison, Isot. Environ. Healt. S., 2006, 42(3), 217-277.

44 R. D. L. Johnstone, A. R. Lennie, S. F. Parker, S. Parsons, E. Pidcock, P. R. Richardson, J. E. Warren and P. A. Wood, CrystEngComm, 2010, 12, 1065-1078.

45 C. L. Bull, N. P. Funnell, C. J. Ridley, C. R. Pulham, P. L. Coster, J. P. Tellam and W. G. Marshall, CrystEngComm, 2019, 21, 5872-5881.

46 J. Nyman and G. M. Day, Phys. Chem. Chem. Phys., 2016, 18, 31132-31143.

47 C. F. Macrae, I. J. Bruno, J. A. Chisholm, P. R. Edgington, P. McCabe, E. Pidcock, L. Rodriguez-Monge, R. Taylor, J. van der Streek and P. A. Wood, J. Appl. Cryst., 2008, 41, 466-470.

48 S. A. Rivera, D. G. Allis and B. S. Hudson, Cryst. Growth Des., 2008, 8(11), 3905-3907.

49 R. I. Smith, S. Hull, M. G. Tucker, H. Y. Playford, D. J. McPhail, S. P. Waller and S. T. Norberg, Rev. Sci. Instrum., 2019, 90, 115101. 
Crystallisation at pressure overcomes the effect of isotopic polymorphism in the methylpyridine pentachlorophenol co-crystal. Though the hydrogenated Cc polymorph can only be obtained at pressure, it is stable on recovery to ambient conditions. 

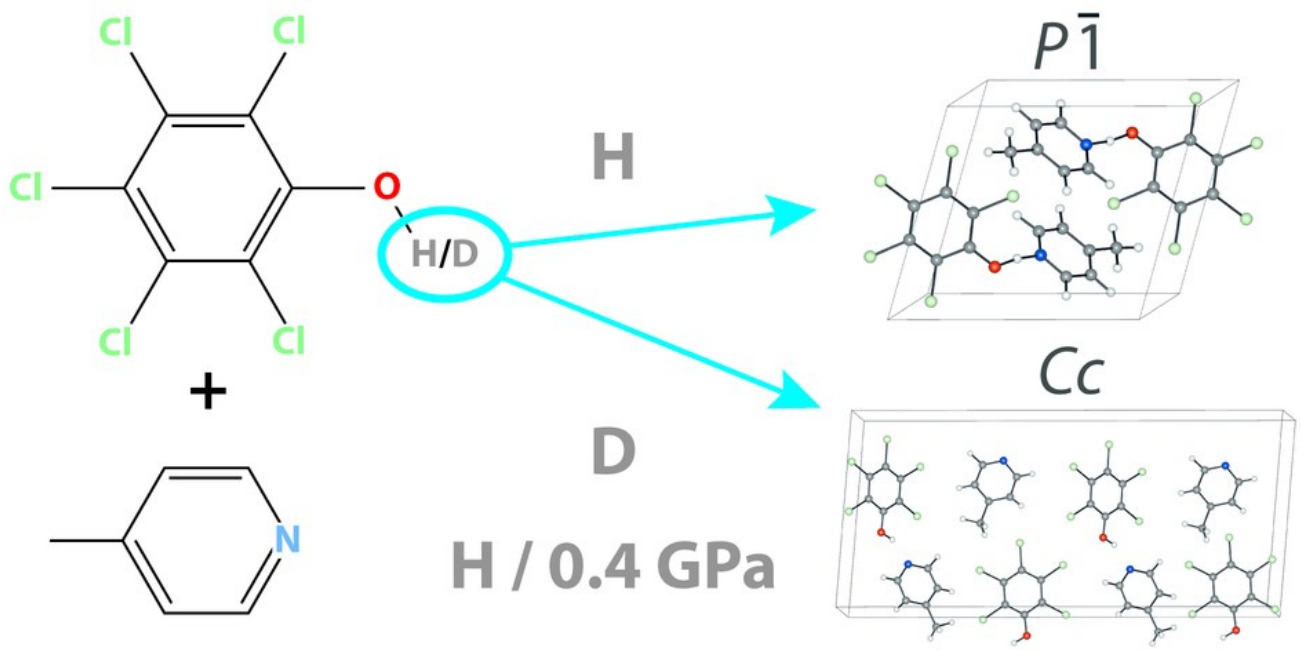

$80 \times 40 \mathrm{~mm}(300 \times 300$ DPI $)$ 